\title{
La imagen construida a partir del discurso. Foro inseguridad vs seguridad: el caso del cierre de calles en Ciudad Juárez
}

NIBIA VERÓNICA MÉNDEZ ARANDA

> Universidad Autónoma de Ciudad Juárez, Ciudad Juárez, México.nibiav79@gmail.com

Universidad de Valparaíso

Facultad de Arquitectura

Revista Márgenes

Espacio Arte Sociedad

La imagen construida a partir del discurso. Foro inseguridad vs seguridad:

el caso del cierre de calles en Ciudad Juárez

Septiembre 2014 Vol $11 \mathrm{~N}^{\circ} 14$

Páginas 7 a 14

ISSN elec. 0719-4463

ISSN imp. 0718-4034

Recepción: mayo 2014

Aceptación: agosto 2014

\section{RESUMEN}

En atención a los incrementos de homicidios registrados por el INEGI y CONAPO en Ciudad Juárez, Chihuahua, México, durante el 2008 se registró una alza respecto a los años anteriores reportando una estadística de 1.569 ejecuciones, los cuales aumentaron a 2.372 durante el 2008, año donde se alcanzó su máxima representación, seguido del año 2010 con unos 2.218 homicidios y en el 2011 con 2.015 dentro de este territorio. A finales del año de 2007 al 2012, el Plan Estratégico de Ciudad Juárez (asociación civil) convocó a través de la Red de Vecinos a debatir el problema de la inseguridad para dialogar con representantes de instancias gubernamentales, los ciudadanos y líderes de las calles cerradas. Y así analizar la realidad de la frontera con la finalidad de conocer sus derechos y posturas en relación a la inseguridad. El proceso de transformación y apropiación del territorio que tiene como peculiaridad inicial el cercado por iniciativa de los propios vecinos, mediante la instalación de barreras físicas y simbólicas en busca de una mayor seguridad. Esto es una reacción de autodefensa que representa una sensación de miedo por parte de los sujetos que lo habitan al sentirse desprotegidos.

Se presenta un análisis del discurso en base a los relatos expuestos a partir de lo que gente habla en relación con sus sentimientos, apoyado por la semántica de los diferentes textos que analizan su contenido para poder conocer la situación de miedo, inseguridad y violencia de Ciudad Juárez a través de imaginario urbano que se simboliza con el cierre de calles por medio de las diferentes percepciones y respuestas subjetivas de los vecinos e integrantes del foro.

PALABRAS CLAVE

seguridad, inseguridad, cierre de calles, miedo, imaginarios urbanos

\section{The image constructed from speech. Forum insecurity vs security: the case of the closure of streets in Ciudad Juarez ABSTRACT}

In response to increases recorded by the INEGI and CONAPO in Ciudad Juárez, Chihuahua, México, in 2008 homicides recorded one hike over previous years reporting a statistic of 1.569 executions, which increased to 2.372 in 2008 , the year with maximum representation, followed by the year 2010 with 2.218 homicides and in 2011 to 2.015 in this territory was reached.

In late 2007 to 2012, the Strategic Plan of Ciudad Juárez (civil society) convened by the Network Neighborhood to discuss the problem of insecurity for dialogue with representatives of government agencies, citizens and leaders of the closed streets. And analyze the reality of the border in order to know their rights and positions in relation to insecurity. The process of transformation and appropriation of territory, which initial enclosure by the installation of physical and symbolic barriers in search of greater security, was made by the initiative of the neighbors. This is a self-defense reaction representing sense of fear on the part of the subjects who inhabit the feel unprotected.

A critical discourse analysis is presented based on the stories exposed from what people are talking about in relation to their feelings, supported by the semantics of the various texts that analyze their content to know the situation of fear, insecurity and violence Ciudad Juarez through urban imaginary that is symbolized by the closure of streets through the different perceptions and subjective responses from neighbors and members of the forum. KEYWORDS

security, insecurity, road closures, fear, urban imaginary 


\section{L'image construite à partir du discours. Forum insécurité vs sécurité: le cas de la fermeture de rues à Ciudad Juárez RÉSUMÉ}

En attention aux accroissements d'homicides enregistrés par I'INEGI et CONAPO à Ciudad Juarez, Chihuahua, Mexique, pendant 2008 nous avons enregistré une hausse par rapport aux années précédentes qui reportaient une statistique de 1.569 exécutions, à 2.372 pendants 2008, année qui a atteint sa représentation maximale, suivi de l'année 2010 avec 2.218 homicides et 2011 avec 2.015 dans ce territoire.

Entre la fin de l'année 2007 jusqu'a 2012, le Plan stratégique de Ciudad Juarez (association civile) a convoqué à travers le Réseau de Voisins à débattre sur le problème de l'insécurité, pour dialoguer avec des représentants gouvernementaux, des citoyens et des chefs des rues fermées. Pour ainsi analyser la réalité de la frontière afin de connaître leurs droits et positions par rapport à l'insécurité. Le processus de transformation et appropriation du territoire a comme particularité initiale celui de ce renfermé, par l'installation de barrières physiques et symboliques à la recherche d'une plus grande sécurité. Ceci est une réaction d'autodéfense qui représente la sensation de peur par les sujets qui l'habitent, car ils se sentent menacés.

Une analyse du discours se présente sur la base des récits exposées à partir de ce que des gens parlent par rapport à ses sentiments, soutenus par la sémantique des différents textes qui analysent leur contenu pour pouvoir connaître la situation de peur, insécurité et violence de Ciudad Juarez à travers l'imaginaire urbain qui est symbolisé par la fermeture des rues au moyen des différentes perceptions et des réponses subjectives des voisins et des membres du forum.

MOTS CLÉS

sécurité, insécurité, fermeture de rues, peur, imaginaires urbains

\section{INTRODUCCIÓN}

El objetivo del presente artículo pretende conocer ¿Cuáles son las prácticas que se entrecruzan para poder producir el escenario del cierre de calles en Ciudad Juárez?, ¿Cuál es el debate de los ciudadanos y las instituciones gubernamentales en relación a las prácticas del cierre de calles?, interrogantes que se podrán responder mediante un análisis del discurso que genera una imagen de la situación actual dentro del foro de inseguridad vs seguridad.

El del presente trabajo consiste en el análisis de dos asambleas realizadas en las oficinas de Plan Estratégico de Juárez bajo la organización del grupo Red de Vecinos (organización civil), que se complementa por comités de vecinos de Ciudad Juárez, la cual convocó a expertos en el tema y funcionarios públicos a participar. La organización del foro estaba a cargo de un mediador que cedía la palabra en un primer tiempo a los invitados y después en un segundo, a los representantes de los diferentes comités de vecinos.

En cuanto a la intervención del investigador fue en base a una auto observación (Álvarez-Gayou, 2007), considerándose como objetivo de estudio el cierre de calles y la imagen que se construye sobre este proceso urbano en Juárez, analizado a partir de las voces de los actores como habitantes, funcionarios, miembros de organizaciones civiles, entre otros.

Por lo tanto es importante razonar una realidad desde un acercamiento interdisciplinar, como lo es un análisis de un discurso, partiendo de una visión que comprende desde un significado contextual que se muestra en las representaciones sociales, dentro de las comunidades urbanas que nos permiten ser abordadas desde la subjetividad humana, lugar en donde se entreteje la realidad y se construye la visión multidimensional de las sociedades contemporáneas, a través de las acciones y representaciones sociales como lo son la apropiación de los espacios de lo público (Rabotnikof, 2005), iniciativa que se ha ido realizando al cerrar las calles que habitan algunos de los ciudadanos de Ciudad Juárez.
La investigación se divide en tres fases analíticas: la primera es una reflexión que construye una visión teórica del análisis del discurso, herramienta para estudiar el problema social y así comprender los mecanismos de poder que se manejan en torno a esta situación, ya que los actos del cierre de calles de los ciudadanos en Ciudad Juárez, en realidad son actos discursivos.

Un segundo momento comprende un análisis teórico y metodológico cualitativo, ya que se analizan las vivencias de las personas por medio de su naturaleza, posibilidad y límites de conocimiento, lo cual explica un planteamiento a través de la interpretación centrada en un conocimiento adquirido por la realidad exterior creador con el cierre de calles en Ciudad Juárez, el cual se analizará a través de técnicas como el análisis del discurso, la subjetividad individual y colectiva dentro de un imaginario social, a partir de los resultados que se desprenden de la etnografía y la fenomenología que se entretejen con la realidad multidimensional de las sociedades contemporáneas.

En un tercer momento se muestran los resultados obtenidos del foro, considerando a los conferencistas como parte importante para incluirlos en el discurso al igual que a los actores que viven el cierre de calles, considerando su participación dentro del foro lo cual se complementa con discursos de los periódicos locales en relación al análisis.

El artículo contiene una reflexión teórica-práctica, basada en un análisis hemerográfico y de entrevistas en relación a los participantes del foro, datos estadísticos, observación de campo apoyado en la fotografía y reflexiones finales.

\section{PRIMER NIVEL DE ANÁLISIS}

\section{Algunas reflexiones teóricas sobre el análisis del discurso}

El presente estudio se explica desde un análisis del discurso, ya que se estructuró desde el lenguaje como práctica social, colocan- 
do al propio lenguaje como la base del análisis. Se tomó en cuenta desde una visión por medio de dos puntos: la lingüística y su análisis del discurso ya que son disciplinas que se encargan de analizar las relaciones de autoridad, discriminación y control por medio del lenguaje y éstos sólo se pueden centrar en textos, redactados o hablados, que consisten en un teorización, descripción de procesos y estructuras sociales que son las que originan los textos y éstos son las unidades básicas para la comprensión de las prácticas sociales (Wodak, 2003).

De este modo, Michael Meyer (2003:33) asevera que el análisis del discurso es un enfoque que consiste en varios planos y cada uno de ellos demanda una selección de contenido y al igual que Wodak (2003:17) establece una relación entre lenguaje y sociedad y la interdisciplinariedad puede llevarlo a ser descrito por diferentes perspectivas, en relación a los discursos históricos que incluyen elementos socio-psicológico y político e ideológico.

La importancia del análisis del discurso de acuerdo a estos autores, es que está conformado por procesos y estructuras sociales de individuos o de grupos que producen un texto a lo largo de la historia de las comunidades, las cuales están basadas en la ideología, representaciones simbólicas que se relacionan con la cultura, que se entrelaza con las formas y procesos sociales que da como resultado una serie de manifestaciones dentro del mundo social a partir de pensamientos y la realidad.

En relación con lo anterior, Wodak (2003:33) constituye que el análisis del discurso se puede analizar a partir de tres conceptos: el concepto de poder, de historia y el de ideología. Teniendo en cuenta que todo discurso es un objeto históricamente producido e interpretado, situado en el tiempo y espacio, y que las estructuras de dominancia están legitimadas por las ideologías de grupos poderosos (Wodak, 2003:19-20).

Por lo tanto un discurso se puede analizar desde una ideología establecida como algo ya dado, como si ésta misma fuera un efecto de poder dentro de las estructuras dominantes. Entonces para poder analizar un discurso hay que entender que las personas razonan, sienten, desean e imaginan de acuerdo a razones históricas específicas.

Consecuentemente hay que tener en cuenta que el análisis del discurso (Van Dijk, 2003:144) es sólo una perspectiva crítica que puede combinarse con cualquier punto de vista de las humanidades y las ciencias sociales. La finalidad de usar el análisis del discurso como técnica, herramienta y perspectiva teorética (Fairclough, 2003), es para orientarse en cómo se realizó este estudio, evitar desviaciones y centrarse en el problema real que versa sobre el lenguaje visual, corporal, que se analiza a través lenguaje o estas semiosis, primero a través de las teorías y el marco analítico, el método, desde un punto de vista crítico dentro de una relación transdiciplinar.

De acuerdo con lo anterior, la necesidad de llevar a cabo este análisis es porque las relaciones entre la sociedad son tan complejas y existen tantas actividades dentro de ella que para poder entenderlas, se necesita una comprensión teórica de las aserciones básicas de la gramática y de su enfoque desde el análisis lingüístico tomando en cuenta las diferentes perspectivas y opiniones de los participantes, las cuales entretejen visiones desde una realidad subjetiva, que construye un imaginario urbano que nos presenta un mejor enfoque de la situación actual desde una forma interdisciplinar.
SEGUNDO NIVEL DE ANÁLISIS

\section{Una propuesta metodológica del análisis del discurso desde la formación del imaginario y su aplicación en el análisis del cierre de calles en Ciudad Juárez}

El presente estudio tiene como base teórica lo citado por la hermenéutica, que se comprende desde un enfoque a estudiar del texto y el discurso, apoyado en categorías lingüísticas, donde no se hicieron de lado el tema y los contenidos, sino que se tomaron en cuenta las operacionalizaciones esenciales que dependen en su mayoría de estas categorías que conforma el gran corpus del foro, en base a conversaciones, donde se tomaron en cuenta el tipo y forma de argumentación, el simbolismo colectivo, que se refiere al uso del lenguaje, los actores, los turnos de palabra, la coherencia, el orden de las palabras y objeciones (Meyer, 2003).

El presente análisis del discurso se estudió desde la perspectiva de "modelo" (Van Dijk, 1980) el cual es el escenario del foro que se analizó desde el punto de vista del acontecimiento, que es el que construye la representación (semántica) del texto (discursivo). Teniendo en cuenta principalmente las oraciones y proposiciones coherentes, ya que deben estar relacionadas con el modelo usando la teoría de la coherencia referencial, a partir de la conexión de sus elementos (Lozano, Peña-Marín, Abril, 2008), la cual consiste en descifrar la verdad a partir de individuales para derivar una coherencia en conjunto.

En relación con lo anterior y con las estructuras y funciones del discurso de Van Dijk (1980:107), la coherencia está basada en la interpretación individual que se entrelaza con otras frases. El discurso menciona la percepción del miedo vivido; dentro de un análisis de semántica se muestran las experiencias y sentimientos; por último la forma y orden de las palabras nos conforman como acontecimientos sociales vividos en un momento histórico.

Por lo tanto el punto de partida fue lo que la gente habla en un imaginario creado por una situación de inadaptabilidad. Para lo cual se tomaron en cuenta las opiniones y emociones, teniendo presente que es una reconstrucción del mundo intersubjetivo, que parte de la situación del cierre de calles en Ciudad Juárez. Haciéndose formaciones discursivas en torno a los acontecimientos que atentaron contra los pobladores con respecto a los sentimientos vividos.

\section{¿Cuál es la relación de la formación discursiva, lo imaginario y la subjetividad?}

A partir de esto se establece una relación entre las dimensiones de lo discursivo, lo imaginario y la subjetividad mediante el modelo de análisis que nos llevó a la comprensión y entendimiento del discurso a partir de la formación imaginaria que se pretende explicar la realidad que se articula por medio de la interdisciplinariedad para tener una mejor deducción. Donde la realidad es un elemento compuesto de deseo y relaciones vivenciales que producen significaciones discursivas, que se experimentan con lo imaginario (María Schaufler; Leila Passerino, 2011).

Las prácticas discursivas parten de la reflexión de la formación imaginaria (Ver Cuadro 1.), que se analizó por medio del discurso que tiene que ver con el sujeto a través de dichas prácticas que se generan por las producciones del sentido que parten de un emisor quien genera la fabricación de codificación del lenguaje que aborda a un receptor y así de esta forma se funda la formación ideológica que se genera en una falsa o verdadera conciencia (Haidar, 1998). 


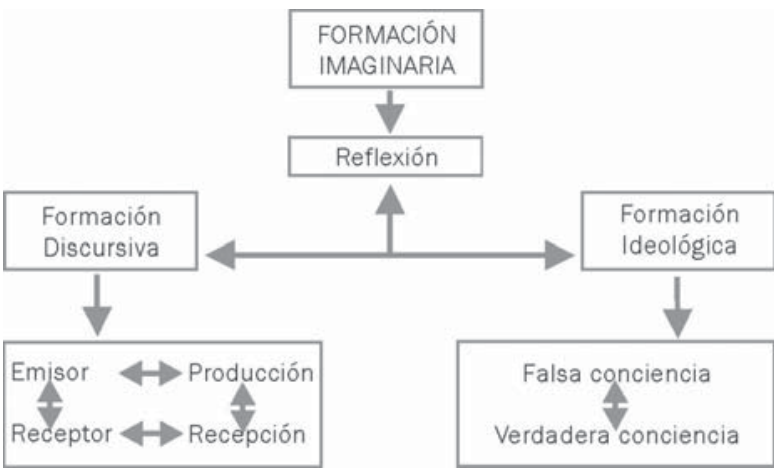

$>$ Cuadro 1. Esquema del análisis del discurso a partir de la formación imaginaria. Elaboración propia

La formación ideológica y lo institucional en relación al análisis del discurso de Julieta Haidar (1998) donde se pretende formarlo, tomando en cuenta primero el estado actual de la cuestión presentada como una "falsa conciencia" ${ }^{1}$, que radica en una indagación y la construcción de sentido, la cual distorsiona la realidad de una manera colectiva para después pasar a una falsa conciencia que es concebida desde la "verdadera conciencia", que se presenta por medio de dicho análisis y así poder encontrar la raíz del conflicto social que nos afecta, a través de una formación discursiva.

Por su parte, María Schaufler y Leila Passerino (2011), abordan lo discursivo en relación al deseo y lo imaginario, que se caracteriza por lo ideológico que oculta las producciones del discurso, llamándolo el disfraz de la percepción directa de la realidad, por lo tanto el imaginario es el que da sentido a las representaciones sociales funcionando como organizador de nuestras relaciones con el mundo y entre nosotros que se producen a partir del deseo y el goce.

Pedro Arturo Gómez (2001:195) hace un análisis de imaginarios sociales y semióticos, como una aproximación para la construcción narrativa de la realidad; esto consiste en entender el funcionamiento de los imaginarios en la sociedad, es decir, lo que se habla dentro de la comunidad en medida y modo de lo que se diga lo cual permite que se procesen representaciones en forma de matrices, que se construyen socialmente y que podemos percibir y aceptarlo como algo real, en donde se puede expresar e inclusive se puede intervenir operativamente de acuerdo a la realidad de cada sistema social.

En relación con lo anterior los procesos discursivos están concernidos con las formaciones imaginarias que derivan de determinados intereses sociales, que se manifiestan, las cuales se conectan con las diferentes maneras de concebir la realidad. Así, la narrativa representa el esquema cognitivo de percepción, que funciona en base a esquemas de fenómenos como el conocimiento, memoria, deseos, sentimientos, conducta, entre otras, que nos permiten interpretar el mundo, en relación con los relatos audiovisuales mediáticos y la legitimación del poder simbólico (Gómez, 2001).

De esta manera, se establece primero una categoría de análisis de los imaginarios sociales en cuanto a la semiótica dentro del universo discursivo: primero instaura que es posible "dar con" y "dar cuenta de" los imaginarios sociales por medio de la materialización discursiva de los imaginarios dentro de textos concretos. Segundo, no es posible una representación sin la intervención o la presencia de los imaginarios y por último hay que tener en cuenta que son los imaginarios los que hacen posible las representaciones.
En este sentido se planteó un análisis de la práctica discursiva a través de un foro de carácter colectivo y socio-cultural, en donde se establecen relaciones entre los diferentes actores y por medio de ellas se llegara a la representación de lugares sociales, los cuales originan una diatriba partiendo de la formación ideológica que gobierna la formación de la acción comunicativa lo cual producirá las variantes del sentido discursivo del análisis del foro.

El ejercicio del discurso se analiza para poder señalar su tópico mediante un proceso de reducción que puede concentrar una secuencia de proposiciones en otra de menor número para poder evolucionar la información como elemento cualitativo central, en base a la estructura superficial que son las oraciones y párrafos (microestructura). Esto a su vez, se refiere a los componentes morfológicos y fonológicos, los cuales constituyen el foro del cierre de calles (Lozano, Peña-Marín, Abril, 2008), (Marín, Morales, 2004), (Fuenmayor, Villasmil, Rincón, 2008).

El presente foro se manejó como un espacio de diálogo y de encuentro de diferentes visiones y experiencias, las cuales forman parte de un proceso que se está realizando con varios comités desde la Red de Vecinos. En esta forma se conforman nuevos discursos que van a servir para lograr la identidad de los vecinos. Ah se planteó el tema de las rejas (cierre de calles en Ciudad Juárez).

El corpus discursivo (Haidar, 1998) se deriva de la formación ideológica del foro y se divide en dos partes:

1. Corpus discursivo I (CDI), representa los agentes, el cual está formado en primer lugar por la asociación civil y representa la visión comunitaria. En este caso es Plan Estratégico de Ciudad Juárez (creador y moderador del foro), en segundo lugar por un representante de los derechos humanos, en tercer lugar es un representante de Desarrollo Urbano del municipio de Ciudad Juárez y por último un licenciado de la Federación Estatal Chihuahuense del Colegio de Abogados en Leyes que representa el enfoque legal.

2. Corpus discursivo II (CDII), son los sujetos (residentes) de Ciudad Juárez, líderes de los diferentes comités de vecinos de comunidades cerradas y abiertas.

\section{TERCER NIVEL DE ANÁLISIS}

\section{Estudio de caso: el cierre de calles en Ciudad Juárez}

La dinámica en la construcción del presente estudio consiste primero en el análisis crítico del discurso en relación a sus visiones primero de los representantes de las instituciones, seguido de las perspectivas presentadas de los residentes de Ciudad Juárez, que en conjunto forman el gran corpus, el cual se pretende analizar por medio de los objetos discursivos que se derivan del contenido del discurso por medio de la intersubjetividad de los sujetos que determinan el presente análisis en base a las variables de inseguridad, seguridad, imaginarios urbanos y cierre de calles.

Para la justificación del foro se mostraron algunas gráficas de encuestas realizadas por Plan Estratégico de Juárez, que exponen los problemas que la ciudadanía considera más importantes, a los participantes que decidieron asistir a la convocatoria de debate sobre el cierre de calles: seguridad vs inseguridad, en Febrero de 2013, los cuales forman el corpus discursivo de esta investigación.

Primero la percepción de inseguridad y violencia de los habitantes de la Ciudad durante el 2011 era más de la mitad (54,6\%) de la población teniendo detectado el desempleo como segundo proble- 
ma seguido de la impunidad. Durante el 2012 esta sensación de inseguridad y violencia disminuyó (32\%), aunque siguió ocupando el primer lugar dentro de la preocupación de los juarenses, seguido de la corrupción y el desempleo.

Otro de los temas que se expusieron en este acto es en relación a los datos expuestos por la mesa de seguridad de Ciudad Juárez (Ver cuadro 2) sobre el robo de autos con violencia que durante el 2010 que fue de 15.800 automóviles y que en trascurso de un año (2011) disminuyó a 13.282 y siguió en una considerable baja durante los meses de Enero a Noviembre del 2012 con 6.400 robos. En relación con este acto de robo pero a comercios con violencia se registraron durante el 2010 la cantidad de 972, cantidad que durante el 2011 descendió considerablemente con una cantidad de reportes de 322 al igual que los registros anteriores sobre el robo de autos descendió considerablemente, pero el robo de tiendas de conveniencia aumentó ya que durante el 2010 se reportaron 2.143 y subió para 2011 con 2.400 , y al igual que los anteriores descendió considerablemente pero hasta el año de 2012 reportando una cantidad de 800 .

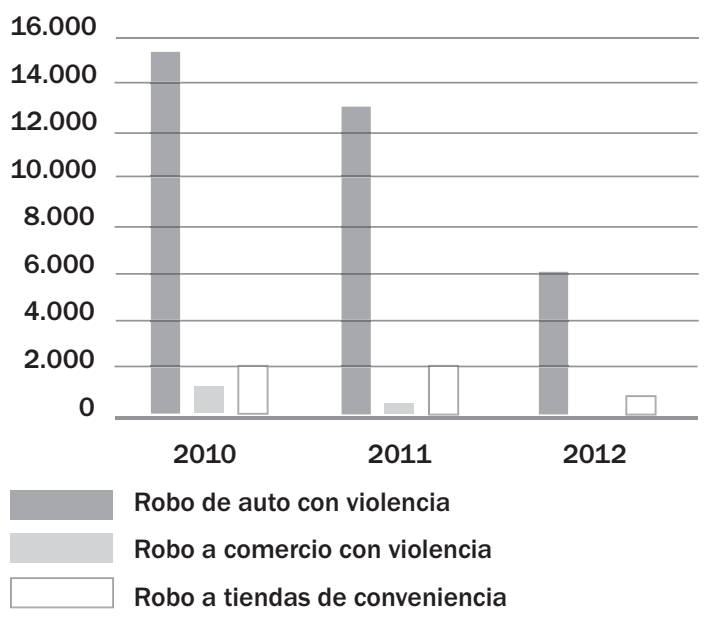

> Cuadro 2. Gráfica con datos de robo de auto y a comercios con violencia y robo a tiendas de conveniencia. Elaboración propia, con datos de la mesa de seguridad de Ciudad Juárez

Todos estos datos nos muestran que los hechos delictivos han ido desapareciendo, pero aún tenemos estadísticas altas de los mismos dentro de la ciudad, por lo tanto la percepción de inseguridad en el sentir de los ciudadanos sigue latente, he aquí la importancia de estudiar el caso del cierre de calles, ya que estos acontecimientos consecuentemente presentan un daño colateral que se simboliza a través del cierre de calles que llevó a cabo la comunidad juarense al interior de sus colonias y fraccionamientos.

Al llevar a cabo el análisis de la narrativa del corpus discursivo I (CDI), que se planteó en relación a la seguridad vs. inseguridad y a los derechos de los ciudadanos se establecieron diversas perspectivas respecto al tema cuando se les cuestionó sobre su postura respecto al cierre de calles como la siguiente:

Uno de los grandes problemas de Ciudad Juárez es el modelo de policía, eso es hasta después, entonces nosotros enfrentamos una cuestión muy contradictoria cómo en Ciudad Juárez se le puede pedir a la gente que no ponga rejas que no se aísle, si la autoridad encargada de la protección de la gente no funciona. 10.500 muertos, homicidios de cinco años son el total de los homicidios que debió de haber habido en Juárez en los próximos 70 años, una autoridad estatal, federal y municipal, que no cumplió dejó a la ciudadanía a medidas de auto defensa (Gustavo de la Rosa, Representante de la Comisión de Derechos Humanos).

En relación con el significado del relato anterior de donde se desprende un reclamo hacia las autoridades y el modelo de policía actual que son las encargadas de brindar seguridad a los ciudadanos. Consecuentemente defiende la postura de los mismos que optaron por una autodefensa justificada desde lo subjetivo en vivencias violentas ocurridas dentro de la ciudad y se combina con el imaginario del miedo colectivo dentro de la comunidad juarense que optaron por una vía de solución inmediata, es el cierre de sus calles en donde los sujetos ante una situación de abandono por parte del gobierno y de violencia depositaron su necesidad de seguridad.

Acto seguido de este cierre de calles, en donde el imaginario del miedo basado en acontecimientos violentos dentro de la ciudad, tiene consecuencias dentro de la comunidad fronteriza juarense, se logra la unión de las personas para encontrar una identidad que les permita hacer comunidad. Como se expresa en el siguiente discurso:

El problema de los juarenses, los fronterizos, los chihuahuenses y los mexicanos, fue muy serio nunca pensamos hubiera aquí en nuestro país un conflicto policial y criminal de la envergadura que vivimos... El problema que nos ocupa es un problema constitucional, el artículo 11 es un artículo muy claro en cuanto a la libertad de tránsito, que no pone mayores limitaciones más que las que determinan los jueces el orden civil y criminal los arraigos y las autoridades administrativas en lo que se refieren a problemas de migración no ponen más límites, entonces es un artículo constitucional que protege, la libertad de los ciudadanos para transitar sin salvoconductos y es donde se encuentra el problema que un ciudadano no puede ingresar a una parte de su territorio si no le autorizan la persona que está en el control de las rejas y no se requiere ni siquiera credencial de elector ni permiso alguno, porque entonces ya violaría ese artículo fundamental (Héctor González, Licenciado de la Federación Estatal Chihuahuense del Colegio de Abogados).

En este sentido se presenta lo que Julieta Haidar (1998:525), acuña como una falsa conciencia en donde lo subjetivo se aborda como principal problema en términos legales principalmente basados en el artículo 11 de la Constitución Política de los Estados Unidos Mexicanos, donde se manifiesta la libertad de tránsito sin salvoconductos, es aquí en donde se genera un discurso de conflicto y sentimientos encontrados entre ciudadanos y vecinos, lo cual distorsiona la situación que se manifiesta a través de un miedo colectivo dentro del territorio donde los ciudadanos se posesionan de las calles por medio de instalación de rejas, piedras, mallas, bardas, troncos, entre otros.

Por lo tanto el imaginario del miedo, reflejado como símbolo en las calles cerradas dentro del territorio, origina las obstrucciones de circulación y de esta manera está violando un artículo fundamental de los otros ciudadanos. Esto representa un problema grave en términos legales, pero el objetivo del problema se manifiesta a través de la percepción de inseguridad y miedo, que consecuentemente los ciudadanos lo depositan en los elementos del cierre de calles y que la solución a este discurso se basa en problemas de seguridad ciudadana. En este sentido también por parte del municipio de Ciudad Juárez se manifestó lo siguiente: 


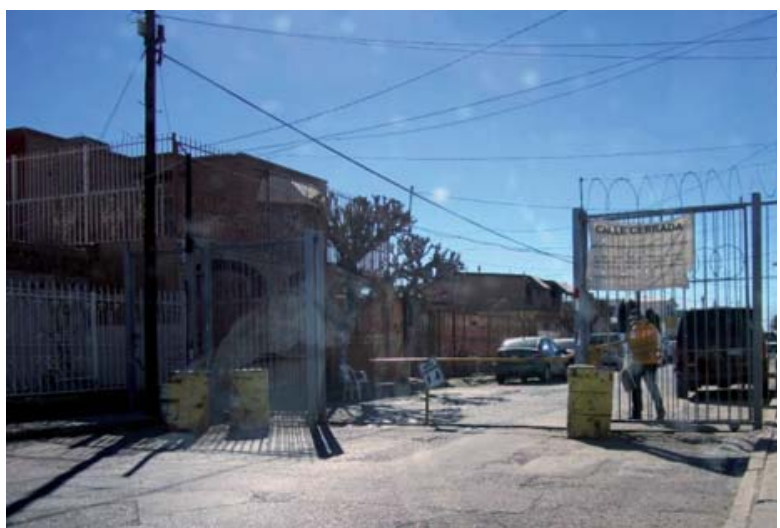

$>$ Figura 1. El imaginario que se representa en fraccionamientos cerrados que realmente se encuentran abiertos

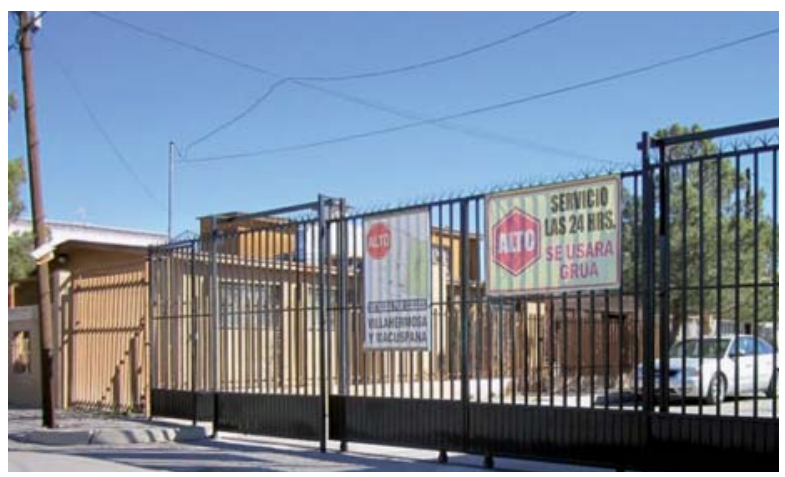

Figura 2. El cierre de calles sólo disminuye los delitos menores

Nos toca conocer muy de cerca tres vertientes, primero el universo que es la inseguridad, pero bien es cierto tenemos grandes intereses el primero son aquellos que tienen interés debido a que el fenómeno de inseguridad que se presentó a finales del 2007 a principios del 2008 se recrudece este fenómeno, una es que los vecinos logran y buscan la manera de tener una seguridad entre comillas porque no es una seguridad total pero cuando menos se sienten un poco seguros (Rodolfo Baylón, Coordinador jurídico de Desarrollo urbano).

Por su parte el Departamento de Desarrollo urbano del municipio de Ciudad Juárez enfatiza el problema principal sobre la inseguridad vivida como se viene repitiendo en el discurso de los diferentes panelistas que se acentúa sobre la subjetividad de las emociones de los ciudadanos en relación con el imaginario de su seguridad que se refleja en el auto-encierro como una forma de auto-exclusión.

De igual forma el siguiente fragmento del análisis del foro por parte de la Directora de Red de Vecinos que se encarga de organizar algunos de los cierres se manifestó en relación a la inseguridad vivida dentro de la ciudad:

La ciudad nos alcanzó con todo y nuestras rejas, con todo y los policías en la calle, con todas las armas con todo $y$ todo el miedo se apoderó de esta ciudad y esto digamos no es nuevo, surge varios años, aparte se agudiza a partir del 2007-2011 ya con las consecuencias que ustedes, bueno que hemos visto (Verónica Corchado, Directora Red de Vecinos).
Por lo tanto se manifiesta por medio de esta declaración, que Ciudad Juárez se ha caracterizado por ser una ciudad donde ocurren sucesos violentos, pero el miedo ha rebasado los sentimientos de los ciudadanos y se agudiza después del año de 2007.

Esta sensación de miedo e inseguridad, en donde los habitantes se sentían desprotegidos, se debe también a declaraciones en los periódicos como la siguiente:

Invade miedo a vecinos de Quintas del Valle. Vecinos del fraccionamiento Quintas del Valle se mostraron temerosos del curso que tomen los operativos federales realizados aquí desde el lunes pasado, mismos que llevaron a la captura de presuntos integrantes de una organización dedicada al "lavado" y traslado de dinero proveniente de operaciones del narcotráfico (El Diario de Ciudad Juárez, 2007:3).

En este sentido la propagación del crecimiento del miedo en base a sucesos violentos dentro de la Ciudad fue acrecentándose, teniendo consecuencias en los sujetos a partir de un sentimiento colectivo que creó el imaginario del miedo por lo que los habitantes de Ciudad Juárez, decidieron cerrar sus calles inclusive en algunos fraccionamientos donde no se haya presentado ningún ataque en contra de la vida de alguno de ellos, teniendo como finalidad su propia protección.

En relación con lo anterior y con la formación ideológica del foro, el corpus discursivo II (CDII) formado por los ciudadanos y algunos líderes de las calles cerradas se expresan de acuerdo a la construcción del discurso de la siguiente manera:

La violencia y la reja por lo menos en mi caso porque tengo las dos opciones de vivir, donde hay reja y no hay reja. La reja a mí no me quitó la violencia de la ciudad, lo único que me tapó fue el tránsito del malhechor pequeño del que se roba la batería, del que entra y golpea a la señora a la casa entonces, es ese el problema que yo veo, limitamos el acceso a nuestros hogares y disminuimos la cantidad de crímenes menores el que aun en mi colonia cerrada si llega alguien con una arma va a pasar entonces, el estar diciendo que la violencia, las rejas no pararon la violencia de los asesinatos (Habitante del Fraccionamiento Florida).

El temor a la violencia de los asesinatos y su percepción de poca seguridad dentro en la ciudad, aún dentro de las calles cerradas queda claro en este discurso, en donde el imaginario consiste en que los ciudadanos quieran atacar los hechos delictivos en base a métodos de auto protección.

De igual forma que los panelistas, los ciudadanos expresaron su desacuerdo con el gobierno mexicano en relación a la seguridad en este sentido:

Considero que una respuesta para la elección que hemos asumido, de vivir en una condición en que la incapacidad del propio Estado nos ha empujado, es de que éste tenga hacia nosotros un enorme agradecimiento, porque en lugar de escoger resolver el problema que se nos presentaba de agresión y violencia hacia la vida nuestra y de nuestras familias tomamos el camino de la auto protección (Presidente del Comité de Vecinos Puesta del Sol).

Por lo tanto se puede entender que no están dispuestos a retirar las rejas, aunque se violen derechos constitucionales, ya que prefieren dentro del imaginario del miedo sentirse más seguros con la auto- 


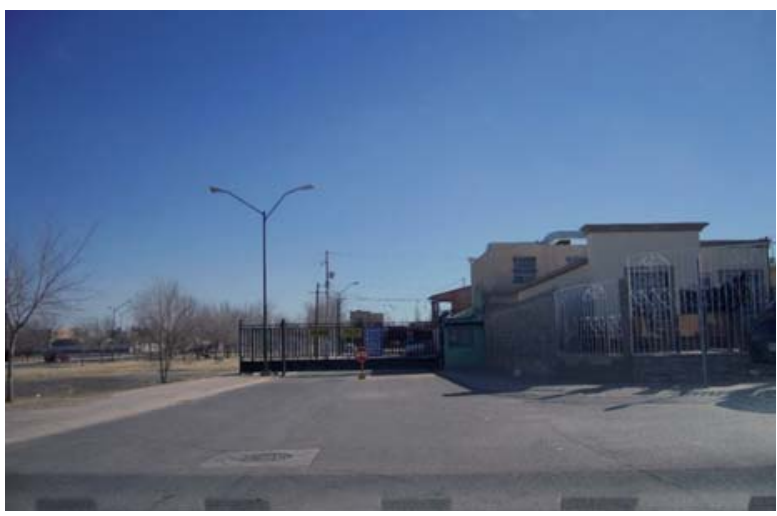

> Figura 3. La apropiación del espacio público resulta en una sensación de seguridad, del apartarse de los 0 tros

protección y depositar su confianza de ellos y sus familias en el cierre de calles. Queda claro que esto es una manera de reclamo ante las instituciones gubernamentales de parte de los ciudadanos por las elevadas cifras de criminalidad que se han venido presentando.

En este orden parte del discurso de seguridad, el imaginario representa la añoranza de espacios públicos seguros en el medio donde se habita:

Yo cuando compré esa casa me encantó que estuviera enfrente de un parque, hace dos años para mi era una maldición ese maldito parque, en una ocasión estuvieron unos sicarios ahi o halcones como les llamen, más de ocho días monitoreándonos a todos, apuntando en una libreta, de ahi empezaron robos, empezaron violencias, todos los vecinos al menos yo personalmente hablé diecisiete veces a la policía y la policía nunca llegó, al último en el 060 me pasaron con el supervisor y la mujer con un acento del sur me dijo y qué quiere que haga si no tenemos patrullas.

Qué bueno que no han sufrido ustedes lo que sufrimos nosotros en su Fraccionamiento y que pueden considerar las rejas, en nuestro fraccionamiento hubo dejen ustedes el robo a baterías, a nosotros nos robaron cinco vehícuIos, me robaron la casa dos veces, se metieron a mi casa cuatro hombres a robar, cuando en otras casas se metieron y abusaron de las personas, violaron hijas, golpearon hombres, fue cuando dije yo, qué sigue de aquí, lo bueno es que somos civilizados y no estamos como en otros países, como el caso ahorita de Guerrero, porque la verdad yo como madre de familia, hubiera conseguido un arma para proteger de que no violaran a mis hijas, las rejas nos han dado tranquilidad, nos han dado unión, entre todos los vecinos (Madre de familia en el Fraccionamiento San Ángel).

En este testimonio la falta de atención a las necesidades de los residentes de Ciudad Juárez, en donde claramente se puede notar que la violencia rebasó los límites y superó al gobierno que es el encargado de brindar seguridad y tranquilidad. Esta comunidad al sentirse desprotegidos, sin armas para responder a tantas agresiones, decidieron auto-segregarse, en base a sus experiencias. En donde la importancia y pérdida del espacio público que se convirtió en zonas de terror un espacio "maldito" ${ }^{2}$ y se hace latente ya que las acciones inmediatas tomadas bajo el imaginario que representa el anhelo de seguridad de todos, decidieron encerrarse junto con estos espacios para poder de esta manera recuperarlos, cuidarlos $\mathrm{y}$ hacer comunidad.
En este orden diferentes ciudadanos, residentes de los fraccionamientos y colonias optaron por expresar sus vivencias en relación a la inseguridad que los llevó a aislarse de los demás:

El problema de la criminalidad rebasó a las autoridades, pero trajo como consecuencia o como secuela, llamémosle la delincuencia desorganizada que son el robo común, el delito común proliferara por indicación, se está hablando de rejas, pero es muy complejo el tema, hay llamémosle dos tipos de rejas una donde está el libre acceso o acceso controlado y el otro donde definitivamente no pueden pasar las personas, es una autoprotección yo siento que la ciudadanía, ante la incapacidad del Estado de proporcionar la seguridad (Ciudadano, Abogado postular).

En relación con lo anterior queda claro que hay quienes dentro de la ciudad han bloqueado absolutamente el paso a toda persona ajena a los residentes de esas comunidades. Existe otro tipo de cierre que posee una entrada y salida controlado donde sólo hay que identificarse para poder accesar a ella. El sentir de los residentes de estas zonas en donde se permite o se prohíbe el acceso es subjetivo a las experiencias vivenciales o a los problemas que hayan surgido entre los vecinos.

De igual manera en todos los relatos de los líderes siguió latente el tema de la seguridad o inseguridad que se vive en la ciudad:

Nuestro fraccionamiento se cerró por la inseguridad, que no se cerró porque vamos a acatar a los sicarios, eso es imposible teníamos que estar todos con armas, de hecho a nosotros nos tocó la experiencia de los mismos policías, encapuchados tapándome los números de las trocas, a cometer delitos y ¿con quién nos dirigimos?, con nadie, porque nadie va hacer nada (Habitante del Fraccionamiento Las Acequias).

También existen discursos en donde el reclamo al modelo de policía, se presentó en todos cada uno con diferentes versiones, y el sentir subjetivo de seguridad que se relacionaba anteriormente con la policía, fue desapareciendo ya que la decepción para los ciudadanos ante su respuesta y acciones como se puede entender en el último discurso. Entonces la comunidad al sentirse abandonada y vulnerable opto por la auto protección creando todo un discurso en relación con lo subjetivo y el imaginario de su seguridad.

\section{REFLEXIONES FINALES}

La búsqueda de la realidad a través representaciones sociales como por ejemplo la defensa del espacio utilizando rejas con distinto tipo de construcción, que se plasman en el análisis del discurso en relación con el imaginario que se producen en la subjetividad humana a partir del goce y el deseo.

En donde el origen o centro del discurso se reduce a partir de la propagación del miedo dentro una colectividad donde la angustia, crea ansiedad, ante un fenómeno atípico como fue el incremento de actos de violencia en Ciudad Juárez, instituyendo una atmósfera de inseguridad.

En base al estudio obtenido por la investigación cualitativa realizada mediante el instrumento del análisis del discurso en relación a la inadaptabilidad ante estos actos delictivos por parte de los ciudadanos crea una ceguera afectiva y propagación del imaginario, el cual tiene como consecuencia una manifestación inmediata dentro de los territorios basados en la sensación de miedo, que se refleja como daño colateral con el cierre de calles dentro de Ciudad Juárez. 
Por lo tanto las ideas que forman el relato con todas estas historias o experiencias vividas, son las que definen y dan sentido a estos espacios cerrados dentro de una ciudad. En el caso de Ciudad Juárez, estas historias están relacionadas con la inseguridad urbana que representa el peligro y las agresiones experimentadas, donde los espacios cerrados o "tomados" representan el miedo de una sociedad, quedando marcados, delimitados y fragmentados.

Las calles de estos espacios pierden el sentido del espacio de lo público (Rabotnikof, 2005) ya que las personas al cerrar se empiezan a apropiar de sus espacios y los hacen suyos a través del control, como algunos lo hacen dentro de los espacios privados.

En relación con lo anterior queda claro que es posible llevar un análisis desde estos espacios cerrados como un "espacio de miedo" desde la subjetividad y el imaginario socio-cultural que representa a un espacio vivido el cual está marcado y delimitado por emociones.

A partir de las sensaciones de miedo, los pobladores experimentan de manera individual y subjetiva y los coloca en un comportamiento natural de legítima defensa, se crea un sentimiento colectivo dentro de una comunidad y se convierte en un hábito permanente que puede ser real o imaginario. Lo cual sirve para satisfacer la necesidad fundamental de seguridad que se encuentra en nuestra base afectiva, como lo expresan los habitantes anteriormente mencionados de los diferentes fraccionamientos y colonias de la Ciudad.

Por lo tanto como resultado final se muestran diferentes expresiones de los habitantes en donde claramente se aprecian estas emociones, las cuales construyen y se representan en el espacio urbano a través de barreras físicas las cuales son el reflejo de un sentido de colectivo, ante una situación de impotencia, que no es más que la suma de todas estas emociones que crean un ambiente de inadaptabilidad, ceguera afectiva y una propagación peligrosa del imaginario (Molinares, Madariaga, 2007), que se exterioriza inmediatamente en el territorio del caso de las calles cerradas que a partir de estas sensaciones-emociones generan un clima de ansiedad, inseguridad y miedo.

Finalmente el surgimiento de la propagación del imaginario se ordenó en base a una secuencia de actos violentos, teniendo sus orígenes en un imaginario individual, basado en discursos individuales de los habitantes de la ciudad. Que derivó en uno colectivo, buscando fortalecer el imaginario del lugar, creando con las calles cerradas en este sentido, una seguridad quizá real para muchos, pero insegura para otros, como se expresan en los discursos analizados de esta investigación de algunos de los habitantes de Ciudad Juárez.

\section{BIBLIOGRAFÍA}

ÁLVAREZ-GAYOU, J. L. (2007) Cómo hacer investigación cualitativa. Fundamentos y metodología. México: Editorial Paidós Mexicana, S. A.

\section{CONSTITUCIÓN POLÍTICA DE LOS ESTADOS UNIDOS MEXICANOS.}

DELUMEAU, J. (2002) EI miedo en Occidente (Siglos XIV-XVIII). Una ciudad sitiada. Madrid: Taurus.

DIJK, T. A. (2003) La multidisciplinariedad del análisis crítico del discurso: un alegato en favor de la diversidad. En R. W. Meyer, Métodos de análisis crítico del discurso, pp. 143176. Barcelona: Gedisa.
(1980) Macroestructures. An interdisciplinary study of global structures in discourse, interaction, and cognition. Hillsdale New Jersey: Lawrence Erlbaum Associates.

(1980) Estructuras y funciones del discurso. Madrid: Cátedra.

EL DIARIO DE CIUDAD JUÁREZ sección A, p. 3-8 de Febrero de 2007.

FAIRCLOUGH, N. (2003) El análisis crítico del discurso como método para la investigación en ciencias sociales. En R. W. Meyer, Métodos de análisis crítico del discurso (pp. 179204). Barcelona: Gedisa.

GÓMEZ, P. A. (2001) Imaginarios sociales y análisis semiótico. Una aproximación a la construcción narrativa de la realidad. Cuaderno de la Facultad de Humanidades y Ciencias Sociales. Universidad Nacional de Jujuy, San Salvador, pp. 195-209.

HAIDAR, J. (1998) Análisis del discurso. En J. G. Cáceres, Técnicas de investigación en sociedad, cultura y comunicación, p. 525. México: Pearson Educación.

LOZANO, J.; PEÑA-MARÍN, C.; ABRIL, G. (2008) Análisis del discurso. Hacia una semiótica de la interacción textual. Madrid: Ediciones Cátedra.

MEYER, M. (2003) Entre la teoría, el método y la política: la ubicación de los enfoques relacionados con el ACD. En R. W. Meyer, Métodos de análisis crítico del discurso (pp. 35-59). Barcelona: Gedisa, S.A.

RABOTNIKOF, N. (2005) En busca de un lugar común. El espacio público en la teoría política contemporánea. México: UNAM.

SCHAUFLER, Ma .; PASSERINO, L. (2011) Aportes para pensar lo imaginario y lo discursivo en las identificaciones políticas. XV Jornadas Nacionales de Investigadores en Comunicación, p. 12.

WODAK, R. (2003) De qué trata el análisis del discurso (ACD). Resumen de su historia, sus conceptos fundamentos y sus desarrollos. En R. W. Mayer, Métodos de análisis crítico del discurso, pp. 17-33. Barcelona: Gedisa.

\section{SECCIÓN HEMEROGRÁFICA}

El Diario de Ciudad Juárez

\section{NOTAS}

1 Vista como una consecuencia a partir de la formación de una ideología que envuelve acontecimientos históricos, sociales y culturales.

2 Expresión usada por los líderes de las colonias en donde se presentan cierres de calles, al referirse a espacios como los parques, plazas, lotes baldíos, entre otros, que quedaron dentro de algunos cierres, inclusive de las mismas calles. 